\title{
The performance of four molecular methods for the laboratory diagnosis of congenital toxoplasmosis in amniotic fluid samples
}

\author{
Leandro Emídio Teixeira ${ }^{[1]}$, Kelly Aparecida Kanunfre ${ }^{[1],[2], ~ P a u l o ~ T a d a s h i ~ S h i m o k a w a ~}{ }^{[1]}$, \\ Lília Spaleta Targa ${ }^{[1]}$, Jonatas Cristian Rodrigues ${ }^{[1],[2]}$, Wilson Domingues ${ }^{[1]}$, \\ Lidia Yamamoto ${ }^{[1]}$ and Thelma Suely Okay ${ }^{[1]}$
}

[1]. Laboratório de Soroepidemiologia e Imunobiologia, Instituto de Medicina Tropical de São Paulo, Universidade de São Paulo, São Paulo, SP. [2]. Laboratório de Imunologia, Departamento de Doenças Infecciosas e Parasitárias, Faculdade de Medicina, Universidade de São Paulo, São Paulo, SP.

\begin{abstract}
Introduction: Toxoplasmosis may be life-threatening in fetuses and in immune-deficient patients. Conventional laboratory diagnosis of toxoplasmosis is based on the presence of $\operatorname{IgM}$ and IgG anti-Toxoplasma gondii antibodies; however, molecular techniques have emerged as alternative tools due to their increased sensitivity. The aim of this study was to compare the performance of 4 PCR-based methods for the laboratory diagnosis of toxoplasmosis. One hundred pregnant women who seroconverted during pregnancy were included in the study. The definition of cases was based on a 12-month follow-up of the infants. Methods: Amniotic fluid samples were submitted to DNA extraction and amplification by the following 4 Toxoplasma techniques performed with parasite B1 gene primers: conventional PCR, nested-PCR, multiplex-nested-PCR, and real-time PCR. Seven parameters were analyzed, sensitivity (Se), specificity (Sp), positive predictive value (PPV), negative predictive value (NPV), positive likelihood ratio (PLR), negative likelihood ratio (NLR) and efficiency (Ef). Results: Fiftynine of the 100 infants had toxoplasmosis; $42(71.2 \%)$ had IgM antibodies at birth but were asymptomatic, and the remaining 17 cases had non-detectable IgM antibodies but high IgG antibody titers that were associated with retinochoroiditis in $8(13.5 \%)$ cases, abnormal cranial ultrasound in $5(8.5 \%)$ cases, and signs/symptoms suggestive of infection in $4(6.8 \%)$ cases. The conventional PCR assay detected 50 cases (9 false-negatives), nested-PCR detected 58 cases ( 1 false-negative and 4 falsepositives), multiplex-nested-PCR detected 57 cases ( 2 false-negatives), and real-time-PCR detected 58 cases ( 1 false-negative). Conclusions: The real-time PCR assay was the best-performing technique based on the parameters of Se (98.3\%), Sp (100\%), PPV (100\%), NPV (97.6\%), PLR ( $\infty)$, NLR (0.017), and Ef (99\%).
\end{abstract}

Keywords: Congenital toxoplasmosis. Congenital infection. Molecular diagnosis. PCR. Quantitative PCR. Real-time PCR.

\section{INTRODUCTION}

Toxoplasmosis is a worldwide endemic protozoan disease that is acquired mainly through infected meat. It is considered a major health problem in pregnant women and in immunecompromised patients. Primary maternal Toxoplasma infection may be health-threatening for the fetus, or may even cause death in utero, depending on the date of transmission, parasite burden, parasite genotype, and host susceptibility. The reactivation of a latent infection under immune-deficiency conditions, such as acquired immunodeficiency syndrome (AIDS) and organ transplantation, can cause fatal toxoplasmic encephalitis.

Address to: $\mathrm{Dr}^{\mathrm{a}}$ Thelma Suely Okay. Lab. de Soroepidemiologia e Imunobiologia/ IMTSP/USP. Av. Doutor Enéas Carvalho de Aguiar 470, Prédio II, $4^{\circ}$ andar, Cerqueira César, 05403-000 São Paulo, SP, Brasil.

Phone: 5511 3061-7022; Fax: 5511 3061-8270

e-mail: thelma.okay@usp.br

Received 3 May 2013

Accepted 27 September 2013
Toxoplasmosis is also an important cause of chorioretinitis, especially in individuals with an impaired immune system. In these conditions, a rapid and accurate diagnosis is required to enable initiation of anti-parasitic treatment as early as possible to improve the prognosis ${ }^{1}$.

While conventional laboratory diagnosis is based on serology parameters, such as the presence of IgM and IgG antiToxoplasma antibodies and, to a minor extent, on the parasite isolations in mice or in cell culture that are performed only in reference centers, molecular techniques, such as polymerase chain reaction (PCR), have recently emerged as alternative tools to improve the laboratory diagnosis of toxoplasmosis due to their increased sensitivity ${ }^{2-4}$. Therefore, the diagnosis of clinically relevant congenital toxoplasmosis now relies on molecular techniques, although these techniques are prone to performance variation due to a large diversity of target genes and primers 5 , in addition to factors associated with the proficiency of laboratories in the optimization of PCR conditions ${ }^{2}$.

Recently, the superiority of quantitative amplification methods over older techniques, such as one-round and nestedPCR, has been reported ${ }^{2-4}$, but there is a lack of information regarding the performance of such methods in Brazil. 
In an attempt to take the first step toward a consensus on the best molecular technique for diagnosing congenital toxoplasmosis in Brazilian patients, we compared 4 PCR-based methods performed with parasite $\mathrm{B} 1$ gene primers, varying from conventional systems containing 1 or 2 rounds of amplification to real-time PCR, to determine the best-performing technique. The 4 amplification methods were based on B1 gene primers because in previous experiments analyzing a significant number of amniotic fluid samples of Brazilian women, B1 gene primers performed better than rep529 and ribosomal deoxyribonucleic acid (rDNA) primers ${ }^{5}$.

\section{Study design}

One hundred pregnant women receiving prenatal care at public hospitals of the State of São Paulo and presenting with toxoplasmosis seroconversion, i.e., the presence of positive immunoglobulin $\mathrm{M}(\mathrm{IgM})$ and significant titers of immunoglobulin G (IgG) anti-Toxoplasma gondii antibodies, were screened by commercial immune enzyme assays, chemiluminescence, or immunofluorimetry, depending on local availability. This initial serology was confirmed by indirect immunofluorescence or IgM capture according to local laboratory availability and expertise. The IgG avidity test was used in 16 of the 100 patients. Exclusion criteria were inadequate amniotic fluid samples, due to mixture with either blood or meconium, or sample insufficiency. Among the 100 pregnant women, 23 underwent amniocentesis during the $16^{\text {th }}$ week of gestation, 20 during the $17^{\text {th }}$ week, 18 during the $18^{\text {th }}$ week, 13 during the $19^{\text {th }}$ week, 5 during the $20^{\text {th }}$ or $21^{\text {st }}$ weeks, 6 during the $22^{\text {nd }}$ week, 5 during the $23^{\text {rd }}$ week, 3 during the $24^{\text {th }}$ week, and 2 during the $25^{\text {th }}$ week of pregnancy. The procedure was performed after the women signed informed consent forms (Institutional Ethics Committee protocol number 0715/2011). All 100 women received treatment with $3 \mathrm{~g} /$ day of spiramycin, which was prescribed as soon as the attending physician received the serology results and continued until 1 week before the scheduled date of delivery to minimize the risk of bleeding. Patients were informed of the risks of amniocentesis, as well as the nucleic acid procedures that would be performed in the context of the research, and the participants consented to all subsequent testing.

\section{Case definition}

A case of congenital toxoplasmosis was confirmed when the newborn/infant had anti-T. gondii $\operatorname{IgM}$ antibodies and/or high IgG antibody titers (significantly higher than the maternal titers at birth and $>300 \mathrm{IU} / \mathrm{ml}$ in immune enzyme assays at any time point) at birth or at some point during the 12-month follow-up. In the absence of positive IgM antibodies, high IgG antibody titers should be associated with signs and symptoms suggestive of toxoplasmosis and/or cranial ultrasound and/or eye fundus abnormalities. These complementary tests, together with serology, were repeated every 3 months during the follow-up.

\section{METHODS}

\section{DNA extraction and assessment of DNA concentration}

Deoxyribonucleic acid (DNA) extraction from amniotic fluid samples was performed with a commercial kit (QIAamp DNA mini kit, QIAGEN Inc., Valencia, California, USA) according to the manufacturer's instructions. DNA concentration was estimated by ultraviolet (UV) spectrophotometry at $260 \mathrm{~nm}$ (Nanodrop 1000, Thermo Scientific, USA).

\section{Amplifications}

Prior to the specific Toxoplasma gondii amplifications, all DNA samples yielded positive results with human betaactin primers ${ }^{6}$, confirming that there were no PCR inhibitors present. Toxoplasma gondii amplifications were performed in a total volume of $25 \mu \mathrm{l}$, consisting of $100 \mathrm{ng}$ of amniotic fluid sample DNA, $200 \mu \mathrm{M}$ of dNTP, $0.2 \mu \mathrm{M}$ of primers, $2.0 \mathrm{mM}$ of $\mathrm{MgCL}_{2}$, and 1.25units of Taq DNA polymerase (GE Healthcare, USA), following stringent procedures to minimize the risk of carryover $^{7}$. A total of 40 cycles/round were performed, with annealing temperatures of $55^{\circ} \mathrm{C}$ for the outer primer pair JW62/ JW63 and $62^{\circ} \mathrm{C}$ for the inner primer pair B22/B23, generating amplification products of 286 and $115 \mathrm{bp}$, respectively ${ }^{5}$. The DNAPCR was performed with primers JW62/JW63; the nested-PCR was performed with primers JW62/JW63 and B22/B23; and the multiplex-nested-PCR was performed with the same JW62/JW63 and B22/B23 primers used for T. gondii detection and other three primer pairs for the simultaneous detection of cytomegalovirus (CMV), herpes simplex virus (HSV), and Epstein-Barr virus $(\mathrm{EBV})^{8,9}$. The composition of the parasite B1 gene primers was: JW63-GCACCTTTCGGACCTCAACAACCG; JW62-TTCTCGCCTCATTTCTGGGTCTA C; B22AACGGGCGAGTAGCACCTGAGGAGA; and B23TGGGTCTACGTCGATGG ATGACAACT. The fourth amplification procedure was a 40-cycle real-time PCR with primers B22/B23. The concentrations of primers and template DNA were the same as those used for the conventional amplifications, and the master mix was prepared with a commercial kit (QuantiFast SYBR Green PCR, QIAGEN). Cycles were performed at $95^{\circ} \mathrm{C}$ for 15 seconds at an annealing temperature of $62^{\circ} \mathrm{C}$ for $50 \mathrm{sec}$, ending with temperatures between 82 and $83^{\circ} \mathrm{C}$ on the melting curve. The slope was -3.54 ; the efficiency was in the range of $92-100 \%$; and the detection limit was 1 parasite in $70 \%$ and 10 parasites in $100 \%$ of experiments (Thermocycler Step One Plus, Applied Biosystems, Foster City, California, USA). Samples were tested in triplicate with real-time PCR, but results were considered only in a binary manner (positive or negative).

\section{RESULTS}

All 100 women in the study had positive IgM and IgG antiT. gondii antibodies as assessed by screening serology and also by a confirmatory test. Unfortunately, the IgG avidity test results could not be used to aid the laboratory diagnosis because these data were only available for 16 of the 100 patients, and the percent avidity was inconclusive in 10 of these 16 cases (data not shown).

The assessment of newborns by clinical, serological, and complementary exams and the monitoring of infants until the age of 12 months revealed that among the 100 total cases, 59 had confirmed toxoplasmosis; 42 (71.2\%) newborns had IgM 
antibodies at birth but were asymptomatic, and the remaining $17(28.8 \%)$ newborns had high $\mathrm{IgG}$ titers $(>300 \mathrm{IU} / \mathrm{ml})$ in the serology tests. These high titers were associated with chorioretinitis in $8(13.5 \%)$ infants; abnormal cranial ultrasound in 5 (8.5\%) infants; and signs/symptoms suggestive of infection, such as marked jaundice, hepatomegaly, and splenomegaly that regressed after treatment, in $4(6.8 \%)$ infants. Among the 59 confirmed cases, 50 were positive based on conventional PCR (9 false-negative results and no false-positive results), 58 based on nested-PCR ( 1 false-negative and 4 false-positive results),
57 based on multiplex-nested-PCR ( 2 false-negative results and no false-positive results), and 58 based on real-time PCR (1 false-negative and no false-positive results).

Parameters of sensitivity (Se), specificity ( $\mathrm{Sp}$ ), positive predictive value (PPV), negative predictive value (NPV), positive likelihood ratio (PLR), negative likelihood ratio (NLR) and efficiency (Ef) of the 4 amplification techniques were analyzed based on the confirmation of positive and negative cases according to their clinical and laboratory evolution until the age of 12 months. The 7 analyzed parameters are displayed in Table 1.

TABLE 1 - The performance of 4 PCR-based techniques for the molecular diagnosis of congenital toxoplasmosis.

\begin{tabular}{|c|c|c|c|c|c|c|c|}
\hline Technique & $\begin{array}{c}\mathrm{Se} \\
(95 \% \mathrm{CI})\end{array}$ & $\begin{array}{c}\mathrm{Sp} \\
(95 \% \mathrm{CI})\end{array}$ & $\begin{array}{c}\text { PPV } \\
(95 \% \mathrm{CI})\end{array}$ & $\begin{array}{c}\text { NPV } \\
(95 \% \mathrm{CI})\end{array}$ & PLR & NLR & $\operatorname{Ef}(\%)$ \\
\hline \multirow[t]{2}{*}{ Conventional PCR } & 84.7 & 100.0 & 100.0 & 82.0 & $\S$ & 0.15 & 91.0 \\
\hline & $(0.73-0.93)$ & $(0.91-1.00)$ & $(0.94-1.00)$ & $(0.69-0.91)$ & & & \\
\hline Nested PCR & 98.3 & 90.2 & 93.5 & 97.4 & 10.07 & 0.018 & 95.0 \\
\hline \multirow[t]{2}{*}{ Multiplex-nested-PCR } & 96.6 & 100.0 & 100.0 & 95.3 & $\S$ & 0.034 & 98.0 \\
\hline & $(0.88-0.99)$ & $(0.91-1.00)$ & $(0.94-1.00)$ & $(0.84-0.99)$ & & & \\
\hline \multirow[t]{2}{*}{ Real-time PCR } & 98.3 & 100.0 & 100.0 & 97.6 & $\S$ & 0.017 & 99.0 \\
\hline & $(0.91-0.99)$ & $(0.91-1.00)$ & $(0.94-1.00)$ & $(0.87-0.99)$ & & & \\
\hline
\end{tabular}

Se: sensitivity; Sp: specificity; PPV: positive predictive value; NPV: negative predictive value; PLR: positive likelihood ratio; NLR: negative likelihood ratio; Ef: efficiency; PCR: polymerase chain reaction; §: infinite; CI: confidence interval.

\section{DIscussion}

The effectiveness of different target genes and primers in the amplification of $T$. gondii DNA in different types of biological samples has long being evaluated in the literature; however, there is a lack of consensus on this topic, at least in non-European countries, and performance is strongly dependent on the proficiency of laboratories in the optimization of the $\mathrm{PCR}^{2,5}$.

Reischl et al. ${ }^{10}$, in a European study, used real-time PCR (LightCycler) with rep529 and B1 gene primers to evaluate 51 Toxoplasma gondii-positive amniotic fluid samples and found a 10-100-fold higher sensitivity for the PCR assay that targeted the 529-bp repeat element of Toxoplasma gondii.

Edvinsson et al. ${ }^{11}$, also in Europe, investigated whether realtime PCR could be used to monitor parasitemia in the blood of a transplant recipient presenting with toxoplasmosis. The results demonstrated that detection of the 529-bp repeat element increased the diagnostic sensitivity and accuracy compared with the assessment of the B1 gene only. These authors also added an internal amplification control that did not apparently affect the
PCR performance and was useful for monitoring PCR inhibition by non-specific DNA in the LightCycler instrument.

One of the first Brazilian studies on the molecular diagnosis of congenital toxoplasmosis was conducted by Vidigal et al. ${ }^{12}$ in 2005. The authors analyzed 86 amniotic fluid samples from women who seroconverted during pregnancy in the State of Minas Gerais. Samples were inoculated in mice and submitted to T. gondii DNA amplification by a nested-PCR. Newborns were also evaluated by $T$. gondii serology, skull x-ray, transfontanel ultrasound, fundoscopic examination, and lumbar puncture. PCR was positive in 7 cases and negative in 79 (7/86 or $8.1 \%)$ cases. Among the PCR-positive cases, two were negative after mouse inoculation; among the PCR-negative cases, 3 had clinical evidence of toxoplasmosis and 1 was positive after mouse inoculation. PCR showed a sensitivity of $62.5 \%$ and specificity of $97.4 \%$ whereas mouse inoculation had a sensitivity of $42.9 \%$ and a specificity of $100 \%$. At that time, the authors considered PCR to be a promising diagnostic method.

In 2009, Okay et al..$^{5}$ conducted a study in the State of São Paulo, Brazil. They analyzed 467 amniotic fluid samples from women who had seroconverted during pregnancy and found $189(40.5 \%)$ positive samples by one-round amplifications: 
$120(63.5 \%)$ with the $T$. gondii B1 gene, 24 (12.7\%) with the parasite AF146527 sequence (also called rep529), 45 (23.8\%) with both, rep529 and the B1 gene, and none with rDNA (ribosomal DNA). In the same study, 50 previously negative one-round PCR samples were chosen by computer-assisted randomization analysis and were re-tested by a nested-PCR based on the parasite B1 gene, which detected 9 additional cases $(9 / 50$ or $18 \%)$. The authors concluded that there was significant performance variation among one-round and nestedamplification systems and showed that the most sensitive method of those tested was a nested-PCR assay with the outer primers JW62/JW63 and the inner primers B22/B23 from the parasite B1 gene compared with other target genes, such as rep529, and rDNA. However, in those experiments, the rep529 primers and amplification products were not suitable for real-time PCR.

In contrast, some studies reported an increased sensitivity of PCR targeting the rep529 sequence over that of the B1 gene ${ }^{2-}$ ${ }^{4}$; these findings were corroborated by Menotti et al. ${ }^{13}$, who developed a new 5'-nuclease real-time PCR assay targeting the rep529 sequence (TaqMan-rep529-PCR) to diagnose toxoplasmosis and assess treatment follow-up. Initially, these authors analyzed 144 clinical specimens for the presence of $T$. gondii DNA by a polymerase chain reaction-enzymelinked immunosorbent assay (PCR-ELISA) assay targeting the parasite B1 gene (B1-PCR-ELISA) and found 15 positive samples, all of which were from patients with clinically proven toxoplasmosis that were negative by the B1-PCR-ELISA and positive according to the TaqMan- rep529 -PCR. Then, the same authors analyzed 203 consecutive clinical specimens during a 4-month period. The diagnosis of toxoplasmosis in two patients was made only by the TaqMan-rep529-PCR and not by the B1-PCR-ELISA. Additionally, samples from a patient with cerebral and disseminated toxoplasmosis, who had already been tested using a B1 real-time PCR assay, were evaluated by the TaqMan-rep529-PCR in a Light Cycler real-time PCR assay targeting the same repetitive rep529 sequence. The TaqManrep529-PCR assay was able to detect positive samples and had a mean gain of 7.1 following 3.3 amplification cycles compared with the B1 real-time PCR assay. These authors concluded that the 5'-nuclease real-time PCR assay developed for the rep529 sequence was more sensitive than those using the $\mathrm{B} 1$ gene.

However, Wahab et al. ${ }^{14}$ showed that the rep529 element was absent in $4.8 \%$ of human $T$. gondii-positive samples, suggesting that the $\mathrm{B} 1$ gene should be considered a preferred diagnostic target. Nevertheless, the rep529 sequence has been the most used sequence in European studies, including a study that analyzed the performance of 3 molecular methods for the amplification of T. gondii DNA in amniotic fluid samples, namely a commercial kit (nested-PCR) and two in-house methods (a conventional PCR and a real-time PCR). This evaluation was based on a T. gondii DNA serial dilution, 3 amniotic fluid samples inoculated with $T$. gondii at different concentrations, and 33 amniotic fluid samples from a clinical cohort. The T. gondii DNA serial dilution assay showed a much lower sensitivity for the commercial kit than for the two in-house methods. Moreover, of the 12 proven congenital toxoplasmosis cases among the cohort, $91.7 \%$ were detected by the in-house assays, whereas only $50 \%$ were detected by the commercial kit ${ }^{15}$. This lack of sensitivity was attributed to the presence of PCR inhibitors.

In a recent Brazilian study ${ }^{16}$, a real-time PCR (qPCR) targeting the parasite rep529 sequence was positive in $72(48 \%)$ blood samples from 150 infected newborns. Among the infants with active retinochoroiditis, $68 \%$ had positive qPCR results, while positivity was much lower $(29 \%, \mathrm{p}=0.009)$ in the absence of ocular involvement. The authors concluded that the qPCR acted as a prognostic factor and that the positivity of samples was associated with the presence of retinochoroidal lesions, with an odds ratio of 2.8. In that study, it is clear that the qPCR assay was used to evaluate the prognosis of newborns with respect to eye injuries and was not used for the purpose of diagnosis, given that the biological material used was blood. This methodology explains the low (48\%) percentage of newborns with confirmed congenital toxoplasmosis and positive qPCR results, contrasting with the 58/59 confirmed cases $(98.3 \%$ ) detected in the amniotic fluid samples of the present study. Nevertheless, as the target gene (rep529 sequence versus B1 gene) and the biological material (peripheral blood versus amniotic fluid samples) were different, prospective studies comparing real time PCR performed with rep529 primers or B1 gene primers in the same type of biological samples are needed. It is also important to clarify that the rep529 pair of primers tested in our laboratory in the experiments published in $2009^{5}$ are not suitable for realtime PCR, contrasting with the rep529 primers described in that recently published Brazilian report ${ }^{13}$.

In the present study, 100 women who seroconverted during pregnancy were included in the study. Among the 100 suspected cases, 59 were confirmed and 41 were excluded after a 12 -month follow-up during which the infants were clinically re-evaluated and also analyzed by means of serology, as well as other exams, e.g., ocular fundoscopy and cranial ultrasound or computerized tomography in older infants, repeated every 3 months. Amniotic fluid samples were subjected to 4 different molecular tests performed with the parasite B1 gene primers, always by the same technician in the same laboratory facility. Importantly, neither technique was able to detect all 59 confirmed cases in this study. However, it is important to mention that we did not stratify the cases according to gestational age at the time of transmission, and this parameter seems to be a determinant of the positivity found by different molecular methods in the congenital toxoplasmosis model. For example, Sterkers et al. ${ }^{17}$ analyzed the performance of an optimized PCR assay tested on amniotic fluid samples, placenta, and cord blood in a cohort of 344 pregnant women who underwent amniocentesis and whose children were followed for 1 year. The authors analyzed the PCR results according to the gestational age at the time of maternal infection and inferred accurate post-test predictive values for diagnosis. They concluded that despite the persistence of falsenegatives, using a highly accurate molecular diagnostic test and the post-test risk curves, it is possible to predict the actual risk of congenital toxoplasmosis at different gestational ages.

According to the analyzed parameters for the 4 molecular techniques and the TDR (UNICEF, UNDP, World Bank, WHO) recommendations developed to guide laboratory diagnostic procedures, including Nucleic Acid Tests (NATs) ${ }^{18}$, DNA-PCR 
should not be used due to its relatively low (84.7\%) sensitivity. This technique failed to detect 9 positive cases, but it did not yield any false-positive results. The nested-PCR technique had a specificity of $90.2 \%$ and thus did not meet the specificity criterion for laboratory diagnostic procedures (specificity $>95 \%$ ). It also produced 4 false-positive results, possibly caused by carryover, which is a known drawback of this type of amplification, even when performed by experienced individuals ${ }^{7}$. Among the methods that complied with the recommended sensitivity and specificity levels, i.e., the multiplex-nested-PCR and real-time PCR techniques, the multiplex-nested-PCR did not produce any false-positive results but detected 1 fewer case than the nestedPCR technique, even though both techniques were performed with the same Toxoplasma primer pairs. This difference was most likely due to competition for the use of reagents, as there were 4 primer pairs in each multiplex-nested-PCR to allow for simultaneous detection of $T$. gondii, CMV, HSV, and $\mathrm{EBV}^{8,9}$. Among the 4 proposed methods, the real-time PCR technique detected 58 of the 59 possible cases did not produce any falsepositive results and was the best-performing test considering all 7 analyzed parameters, a sensitivity of $98.3 \%$, specificity of $100 \%$, PPV of $100 \%$, NPV of $97.6 \%$, an infinite PLR, a NLR of 0.017 , and an efficiency of $99 \%$, corroborating the findings of previous studies $^{2-4}$ that highlighted the advantages of real-time PCR. Moreover, this technique is able to determine the number of parasites/target gene copy number, enabling follow-up of treated patients, and has a short run time, reducing the risk of carry-over and handling of DNA intercalating substances.

In conclusion, in this study performed on 100 amniotic fluid samples from pregnant women living in the State of São Paulo, the best-performing technique, based on the parameters of sensitivity, specificity, positive and negative predictive values, positive and negative likelihood ratios, and efficiency, was the real-time PCR technique with primers B22/23. In the future, we intend to analyze these same amniotic fluid samples with primers of the parasite rep529 sequence using real-time PCR to compare our results with those of other studies.

\section{CONFLICT OF INTEREST}

The authors declare that there is no conflict of interest.

\section{FINANCIAL SUPPORT}

This work was supported by FAPESP (Fundação de Amparo à Pesquisa do Estado de São Paulo; grant number 2010/15022-1), as well as by CNPq (Conselho Nacional de Desenvolvimento Cientifico e Tecnológico; grant number 2011-0/471479).

\section{REFERENCES}

1. Montoya JG, Remington JS. Management of Toxoplasma gondii infection during pregnancy. Clin Infect Dis 2008, 47:554-566.
2. Sterkers Y, Varlet-Marie E, Cassaing S, Brenier-Pinchart MP, Brun S, Dalle F, et al. Multicentric comparative analytical performance study for molecular detection of low amounts of Toxoplasma gondii from simulated specimens. J Clin Microbiol 2010; 48:3216-3222.

3. Nagy B, Bán Z, Beke A, Nagy GR, Lázár L, Papp C, et al. Detection of Toxoplasma gondii from amniotic fluid, a comparison of four different molecular biological methods. Clin Chim Acta 2006; 368:131-137.

4. Cassaing S, Bessières MH, Berry A, Berrebi A, Fabre R, Magnaval JF. Comparison between two amplification sets for molecular diagnosis of toxoplasmosis by real-time PCR. J Clin Microbiol 2006, 44:720-724.

5. Okay TS, Yamamoto L, Oliveira LC, Manuli ER, Andrade Jr HF, Del Negro GM. Significant performance variation among PCR systems in diagnosing congenital toxoplasmosis in São Paulo, Brazil: analysis of 467 amniotic fluid samples. Clinics 2009; 64:171-176.

6. Nakajima-Iljima S, Hamada H, Reddy P, Kakunaga T. Molecular structure of the human cytoplasmic $\beta$-actin gene: interspecies homology of sequences in the introns. Proc Natl Acad Sci USA 1985; 82:6133-6137.

7. Rolfs A, Schuller I, Finckh U, Weber-Rolfs I. PCR contamination and falsely interpreted results. Editor Arndt Rolfs. PCR: clinical diagnostics and research. Berlin: Springer-Verlag 1992; p. 61-67.

8. Camargo PR, Okay TS, Yamamoto L, Del Negro GM, Lopes AA. Myocarditis in children and detection of viruses in myocardial tissue: implications for immunosuppressive therapy. Int J Cardiol 2011; 148: 204-208.

9. Mendes TM, Oliveira LC, Yamamoto L, Del Negro GMB, Okay TS. EpsteinBarr Virus nuclear antigen-2 detection and typing in immunocompromised children correlated with lymphoproliferative disorder biopsy findings. Braz J Infect Dis 2008; 12:186-191.

10. Reischl U, Bretagne S, Krüger D, Ernault P, Costa J-M. Comparison of two DNA targets for the diagnosis of toxoplasmosis by real-time PCR using fluorescence resonance energy transfer hybridization probes. BMC Infectious Diseases 2003; 3:1-9.

11. Edvinsson B, Lappalainen M, Evengard B, on behalf of the ESCMID Study Group for Toxoplasmosis. Real-time PCR targeting a 529-bp repeat element for diagnosis of toxoplasmosis. Clin Microbiol Infect 2006; 12:131-136.

12. Vidigal PVT, Santos DVV, Castro FC, Couto JCF, Vitor RWA, BrasileiroFilho G. Prenatal toxoplasmosis diagnosis from amniotic fluid by PCR. Rev Soc Bras Med Trop 2002; 35:1-6.

13. Menotti J, Garin Y. J-F, Thulliez P, Sérugue M-C, Stanislawiak J, Ribaud $\mathrm{P}$, et al. Evaluation of a new 5'nuclease real-time PCR assay targeting the Toxoplasma gondii AF146527 genomic repeat. Clin Microbiol Infect 2010; 16:363-368.

14. Wahab T, Edvinsson B, Palm D, Lindh J. Comparison of the AF146527 and $\mathrm{B} 1$ repeated elements, two real-time PCR targets used for detection of Toxoplasma gondii. J Clin Microbiol 2010; 48:591-592.

15. Morelle C, Varlet-Marie E, Brenier-Pinchart MP, Cassaing S, Pelloux H, Bastien P, et al. Comparative assessment of a commercial kit and two laboratory-developed PCR assays for molecular diagnosis of congenital toxoplasmosis. J Clin Microbiol 2012; 50:3977-3982.

16. Costa JG, Carneiro AC, Tavares AT, Andrade GM, Vasconcelos-Santos $\mathrm{DV}$, Januário JN, et al. Real-time PCR as prognostic tool of human congenital toxoplasmosis. J Clin Microbiol 2013; Jun 12. [Epub ahead of print].

17. Sterkers Y, Pratlong F, Albaba S, Loubersac J, Picot M-C, Pretet V, et al. Novel interpretation of molecular diagnosis of congenital toxoplasmosis according to gestational age at the time of maternal infection. J Clin Microbiol 2012; 50:3944-3951.

18. The TDR Diagnostics Evaluation Expert Panel. Evaluation of diagnostic tests for infectious diseases: General principles. Nature Reviews/ Microbiology, Macmillan Publishers Limited, S17-S29, December 2010. 